\title{
Impact of the anatomical location and the number of metastatic lymph nodes on gastric cancer patient's survival
}

\author{
Duchon R, Bernadic M, Jr, Pindak D \\ Department of Surgical Oncology, Slovak Medical School and National Cancer Institute, \\ Bratislava, Slovakia. Michal.Bernadic@nou.sk
}

\begin{abstract}
BACKGROUND: The most serious problem in surgical treatment of gastric cancer includes the area of resection and the extent of lymphadenectomy. The extent of gastric resection is determined by the extent of tumor affection. The aim of radical surgical intervention is to achieve microscopically clear resection line, since R0 resection is the main criterion for the patient's prognosis. Curative surgical resection for gastric cancer includes the lymph nodes dissection. In the treatment of gastric cancer, there are two views on the importance of lymphadenectomy. The Far East considers that operation improves the survival and the Europe considers that surgery is not curative, but it determines the staging and prognosis. There is also a difference in staging systems. The one from East is importance based on the anatomical location of affected lymph nodes, the second from Europe is based on the number of positive lymph nodes.

MATERIALS AND METHODS: This work is a retrospective observational study. In the study cohort, comparing the survival of patients according to different classification systems, depending on the $\mathrm{N}$-stage of disease, 119 patients with gastric adenocarcinoma in clinical stage I to III, i.e. without metastasis, who underwent a radical surgical resection with D2 lymphadenectomy, were enrolled. For the evaluation of the survival versus the time after operation, we used Kaplan-Meier method. To evaluate the correlation between the survival rate and the explanatory variables, Cox regression and Kendall correlation coefficient were used. RESULTS: The median survival, according to different classification systems, depending on the $\mathrm{N}$-stage of the disease, was significantly correlated with the survival for the 6th and 7th editions of TNM classification system for the Japanese classification system, for N-ratio classification system).

The new finding was differentiation of patients in groups N1 vs N2 under the 6th TNM classification $(H R=0.910249)$, also a little differentiation in groups N1 vs N2 according to the classification of N-ratio $(\mathrm{HR}=0.8750926)$ and equally a poor differentiation in the survival in groups N2 vs N3 according to the 7th TNM classification $(\mathrm{HR}=0.881797)$.

The strongest correlation reached the Japanese classification system, but not significantly different from the 6th TNM classification system. In the 7th edition of TNM classification system, we then found the weakest correlation with the survival time, but not significantly different from the previous two.

CONCLUSION: Our retrospective study confirmed the strongest correlation between the patient's prognosis and the anatomic localization of the affected lymph nodes. This correlation was not statistically significant compared to the correlation between patient's prognosis and the number of positive lymph nodes. It leads us to the conclusion that both classification systems are comparable and the difference is statistically insignificant (Tab. 4, Fig. 8, Ref. 16). Text in PDF www.elis.sk.

KEY WORDS: gastric cancer, gastrectomy, lymphadenectomy, staging, prognosis.
\end{abstract}

\section{Introduction}

The most serious problem in surgical treatment of gastric cancer includes the extent of organ resection and the extent of lymphadenectomy.

The extent of gastric resection is determined by the extent of tumor affection. The aim of radical surgical intervention is to

Department of Surgical Oncology, Slovak Medical School and National Cancer Institute, Bratislava, Slovakia

Address for correspondence: M. Bernadic Jr, MD, PhD, Department of Surgical Oncology, Slovak Medical School and National Cancer Institute, Klenova 1, SK-833 10 Bratislava, Slovakia.

Phone: +421.903846184 achieve microscopically clear resection line, since the $\mathrm{R} 0$ resection is the main criterium for the patient's prognosis.

Affection of lymph nodes in gastric cancer is the second most important prognostic factor, after the R classification. Metastatic lymph node involvement depends on the T category and, according to the number of lymph nodes affected, the 5-year survival rate is as well as the favorable prognosis of the patient are decreasing (3). By enlarging the extent of lymphadenectomy there is a higher number of lymph nodes removed. This is affecting the staging because macroscopically negative lymph nodes that are proven positive by histologic examination move the patient to a higher stage of the disease, thus improving the prognosis of patients in the lower stage.

The results of surgical treatment of gastric cancer are improving by an earlier detection of early stages, lower perioperative 
morbidity and mortality, a higher amount of radical resections with a better survival (15). However, the problem of radicality, tactics and techniques of lymph node dissection remains unresolved.

Lymph node dissection is a complete removal of regional lymph drainage. Sufficiently performed lymphadenectomy and thorough processing of the specimen by a pathologist aims to investigate and find the greatest number of lymph nodes. It is essential to determine the staging, prognosis estimation, and decision on further treatment. For the correct staging of the disease, at least 15 lymph nodes must be examined. Currently, the importance of lymphadenectomy for the staging of the disease is not the matter of question. The therapeutic effect of lymphadenectomy is constantly debated.

Curative surgical resection for gastric cancer includes the lymph nodes dissection. In the treatment of gastric cancer, there are two views on the importance of lymphadenectomy. The Far East considers that the operation improves survival and the Europe considers that surgery is not curative, but it determines the staging and prognosis. There is also a difference in the staging systems. The one from East is important based on the anatomical location of affected lymph nodes, the second from Europe is based on the number of positive lymph nodes.

The survival of the patients is evaluated according to different classification systems that are based on different parameters:

\section{TNM staging system (TNM)}

The TNM staging for gastric cancer has been included in the first edition of UICC TNM since 1966. The 6th edition of UICC TNM staging system adopted a number-based system for $\mathrm{N}$-staging that provides an easy and accurate prognostic stratification. The UICC TNM staging system is the global standard to evaluate gastric cancer in different institutions $(1,6)$.

Japanese staging systems for gastric cancer (JSGC) - Its anatomical-based $\mathrm{N}$-staging was established based on analysis of lymphadenectomy effectiveness, and naturally provides a direct surgical guidance (12).

$\mathrm{N}$-ratio staging system - the staging system based on the metastatic lymph node ratio between metastatic and examined lymph nodes (N-ratio) in gastric cancer patients $(2,5,7,8,10,11)$.

The 7th edition of TNM staging system (TNM 2010) - during this study, there was an update in TNM classification to the 7 th edition of 2010 , so we also classified according to this. The main change was in classification of carcinoma of oesophagogastric junction, which now belongs to the carcinoma of oesophagus $(4,13,16)$.

\section{Materials and methods}

Input of clinical and demographic information comes from medical records of patients from the Department of surgical oncology Slovak Medical University (SMU) and National Cancer Institute. There was a total of JCGC. number of 156 patients, who underwent surgery due to gastric cancer in the period from January 2007 to December 2009, including.

Out of this number, we retrospectively analyzed a database of 135 patients, who had undergone a radical elective surgery with D2 lymphadenectomy for stages I-III of gastric adenocarcinoma, which was performed by three trained high-volume surgeons. Stages were diagnosed on the basis of pathological findings. Perioperative/neoadjuvant chemotherapy was not applied.

The primary aim of the study was to investigate the impact on the patient survival rate between the anatomical location of affected lymph nodes and the number of positive lymph nodes. We conducted a subanalysis of survival according to the current staging systems. Depending on the N-stage of disease, 119 patients were enrolled and classified according to 6th TNM and N-ratio classification, 83 according to JCGS and 85 according to 7th TNM 2010. All of them undergone a radical surgery with D2 lymphadenectomy. The present systematic comparison was performed according to the number of examined lymph nodes, histologically positive lymph nodes, their ratio and anatomical location of affected lymph nodes (depending on the location of the primary finding of the stomach). Within each staging systems, patients were divided into the groups N0-N3.

Survival was evaluated by statistical tools "Survival analysis". The starting point was the date of surgery. For the evaluation of the survival versus the time after operation, we used Kaplan Meier method. To evaluate the correlation between the survival rate and the explanatory variables, Cox regression and Kendall correlation coefficient were used. Credibility of the model and the conformity prediction and the actual outcome were tested by $\mathrm{Chi}^{2}$ test.

\section{Results}

\section{Comparison of survival according to different staging systems, depending on the $\mathrm{N}$-stages of the disease}

\section{TNM classification system (6th edition)}

According to the 6th TNM classification, we classified 119 patients. The numbers of patients in individual $\mathrm{N}$-stage and corresponding Median survival time analysis are indicated in the Table 1.

The relationship between the survival time (counted from the time of surgery) and the number of positive lymph nodes was analyzed using the Kaplan-Meier method and is shown in the Figure 1.

Tab. 1. Number of patients, median survival time and confidence interval for each $\mathrm{N}$-stage of 6th TNM staging system.

\begin{tabular}{lcccc}
\hline N-stage of disease & N0 & N1 & N2 & N3 \\
\hline Number of patients & 32 & 35 & 36 & 16 \\
Median survival time (days) & 2050 & 600 & 734 & 309 \\
Brookmeyer-Crowley 95\% CI & $1300-2153$ & $441-1093$ & $516-1111$ & $196-429$ \\
\hline
\end{tabular}

Tab. 2. Number of patients, median survival time and confidence interval for each $\mathrm{N}$-category

\begin{tabular}{lcccc}
\hline N-stage of disease & N0 & N1 & N2 & N3 \\
\hline Number of patients & 21 & 25 & 17 & 13 \\
Median of survival (days) & 1792 & 909 & 545 & 255 \\
Brookmeyer-Crowley 95\% CI & $1268-2153$ & $590-1341$ & $398-1217$ & $206-482$ \\
\hline
\end{tabular}




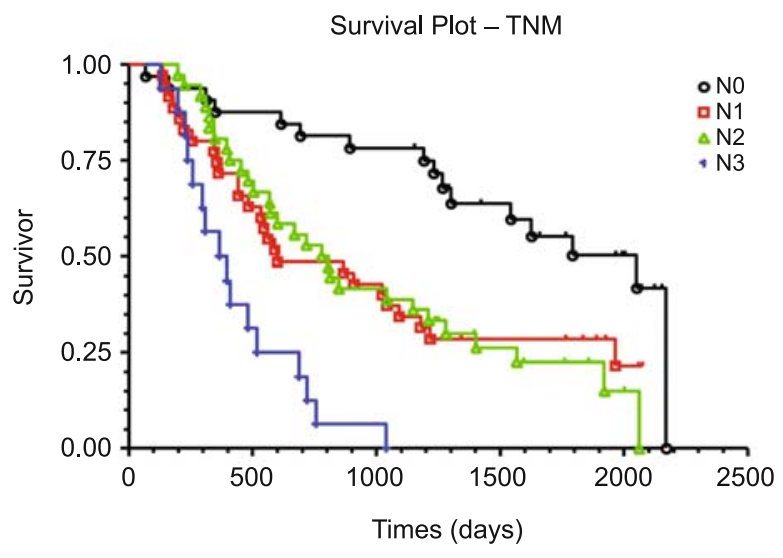

Fig. 1. Survival depending on the $\mathrm{N}$-stage of the disease according to the 6th TNM.

Chi-square test of equivalence mortality $=\mathbf{4 0 . 1 9 2 3 5 2} \mathrm{p}<0.0001$ Comparison of risk categories according to $\mathrm{N}$ (approximate $95 \%$ confidence interval)

N0 vs N1 $=0.430191(0.255011$ to 0.725711$)$

$\mathrm{N} 1$ vs $\mathrm{N} 2=0.910249(0.517242$ to 1.601866$)$

$\mathrm{N} 2$ vs $\mathrm{N} 3=0.355525(0.133008$ to 0.950306$)$

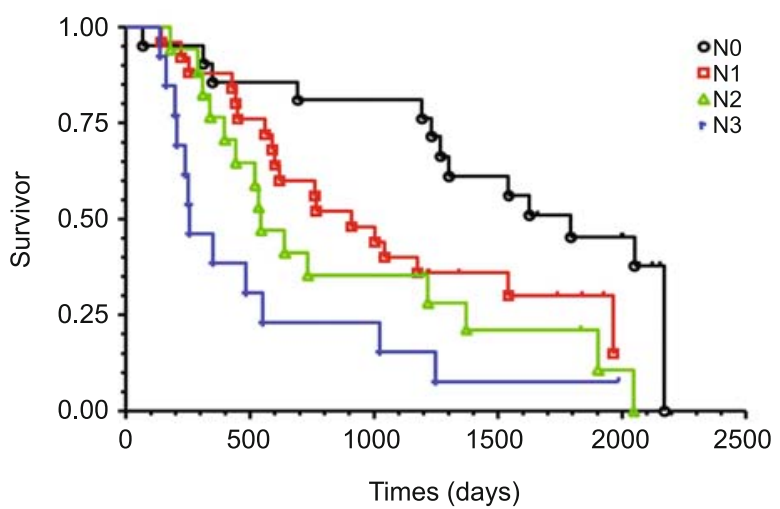

Fig. 2. Survival depending on the $\mathrm{N}$-stage of the disease according to the JSGC.

Chi-square for equivalence of death rates $=19.0073607 \mathrm{p}=\mathbf{0 . 0 0 0 2 7 2 4}$ Comparison of risk categories according to $\mathrm{N}$ (approximate $95 \%$ confidence interval)

N0 vs N1 $=0.5319622(0.2896063$ to 0.9771328$)$

$\mathrm{N} 1$ vs N2 $=0.7045064(0.3299183$ to 1.5044006$)$

N2 vs N3 $=0.5744514(0.1964117$ to 1.6801161$)$

Japanese staging system of gastric cancer

According to the Japanese system, we classified 76 patients, the numbers of patients in each $\mathrm{N}$-stage and the corresponding Median survival time is shown in Table 2.

The evaluation of survival according to the anatomical location of affected lymph nodes according to the time of surgery using the Kaplan-Meier method is shown in the Figure 2.

\section{$N$-ratio staging system}

According to N-ratio staging system, we classified 119 patients, the numbers of patients in each $\mathrm{N}$-stage and the corresponding Median survival time is shown in the Table 3.



Fig. 3. Survival depending on the $\mathrm{N}$-stage of the disease according to the N-ratio staging system.

Chi-square for equivalence of death rates $=22.5222618 \mathrm{p}=\mathbf{0 . 0 0 0 0 5 0 8}$ Comparison of risk categories according to $\mathrm{N}$ (approximate $95 \%$ confidence interval)

N0 vs N1 $=0.5423127(0.2623302$ to 1.1211178$)$

$\mathrm{N} 1$ vs $\mathrm{N} 2=0.8750926(0.358933$ to 2.1335099$)$

$\mathrm{N} 2$ vs $\mathrm{N} 3=0.6235607(0.3087509$ to 1.2593584$)$

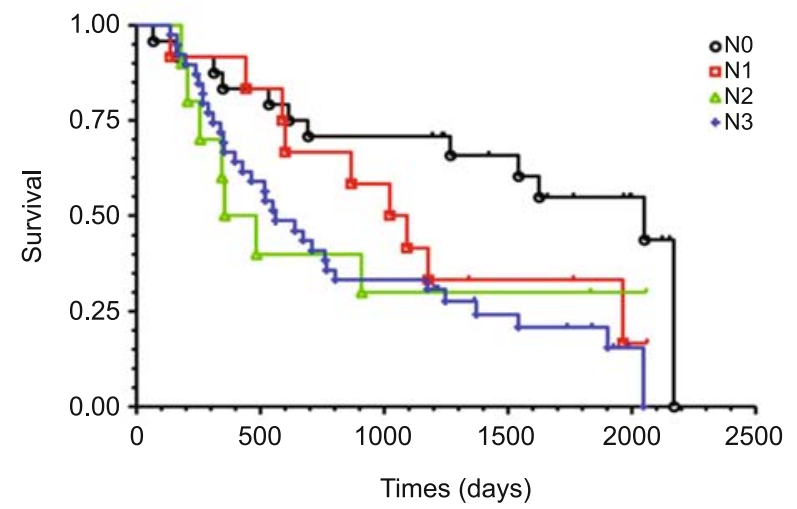

Fig. 4. Survival depending on the $\mathrm{N}$-stage of the disease according to the 7th TNM.

Chi-square for equivalence of death rates $=10.785182 \mathrm{P}=\mathbf{0 . 0 1 2 9}$

Comparison of risk categories according to $\mathrm{N}$ (approximate $95 \%$ confidence interval)

N0 vs N1 $=0.539013(0.251817$ to 1.153755$)$

$\mathrm{N} 1$ vs N2 $=0.758567(0.264318$ to 2.177014$)$

N2 vs N3 $=0.881797(0.346505$ to 2.244025$)$

The evaluation of survival according to the ratio of metastatic and total lymph nodes examined in concordance with the time of surgery using the Kaplan-Meier method is shown in the Figure 3.

\section{The TNM staging system 2010 (7th edition)}

According to the 7 th TNM we classified 85 patients, numbers of patients in each N-category and the corresponding Median survival time is shown in Table 4.

Evaluation of survival according to the ratio of metastatic and total lymph nodes examined in concordance with the time of surgery using the Kaplan-Meier method is shown in Figure 4. 
Tab. 3. The number of patients, median survival time and confidence interval for each $\mathrm{N}$-stage of N-ratio classification system.

\begin{tabular}{lcccc}
\hline N-stage of disease & N0 & N1 & N2 & N3 \\
\hline Number of patients & 32 & 11 & 15 & 61 \\
Median survival time (days) & 2050 & 1179 & 868 & 516 \\
Brookmeyer-Crowley 95\% CI & $1300-2153$ & $441-1765$ & $532-1966$ & $360-670$ \\
\hline
\end{tabular}

Tab. 4. Number of patients, nedian survival time and confidence interval for each $\mathrm{N}$-category of the 7th TNM.

\begin{tabular}{lcccc}
\hline N-stage of disease & N0 & N1 & N2 & N3 \\
\hline Number of patients & 24 & 12 & 10 & 39 \\
Median survival time (days) & 2050 & 1021 & 482 & 560 \\
Brookmeyer-Crowley 95\% CI & $1268-2153$ & $600-1765$ & $255-2058$ & $398-768$ \\
\hline
\end{tabular}

Kendall's tau b correlation coefficient $=-0.333416$

\section{Kendall sequential correlations}

Survival-days vs 6th TNM classification system, observation of a sample of 119 patients

Kendall's tau b correlation coefficient $=-0.333416$

Approximate $95 \% \mathrm{CI}=-0.440676$ to $-0.226155$



Fig. 5. Non-parametric correlation for 6th TNM.

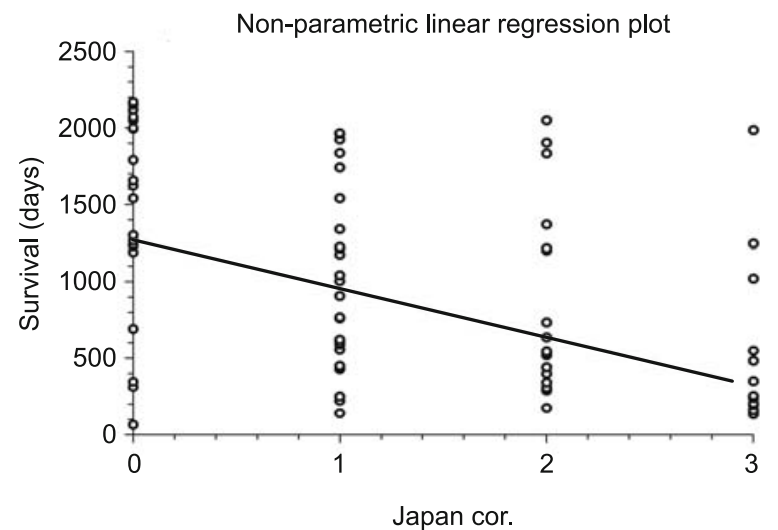

Fig. 6. Non-parametric correlation for JSGC.

A comparison of nonparametric correlation $\mathrm{N}$-stage disease with the survival for the individual staging systems

Correlation 6th TNM staging system vs survival-days (Fig. 5)

Non-parametric linear regression

Correlation survival-days vs 6th TNM

Observation of the sample $=119$

Median slope $(95 \%$ CI $)=-279(-400.666667$ to -150.333333$)$

Y-intercept $=1082$
Correlation JSGC vs survival-days

Non-parametric linear regression (Fig. 6)

Correlation survival-days vs JCGC

Observation of the sample $=76$

Median slope $(95 \%$ CI $)=-316.666667(-461.5$ to -156$)$

Y-intercept $=1272.666667$

Kendall's tau b correlation coefficient $=-0.385601$

Kendall sequential correlations

JSGC vs survival-days

Observation of a sample $=76$

Kendall's tau b correlation coefficient $=-0.385601$

Approximate $95 \% \mathrm{CI}=-0.532939$ to -0.238264

Correlation of $\mathrm{N}$-ratio staging system vs survival-days (Fig. 7)

Non-parametric linear regression

Correlation of survival-days vs N-ratio classification system

Observation of the sample $=119$

Median slope $(95 \% \mathrm{CI})=-247(-342$ to -130.3333333$)$

Y-intercept $=1544$

Kendall's tau b correlation coefficient $=-0.3343553$



Fig. 7. Non-parametric correlation for N-ratio staging system. 


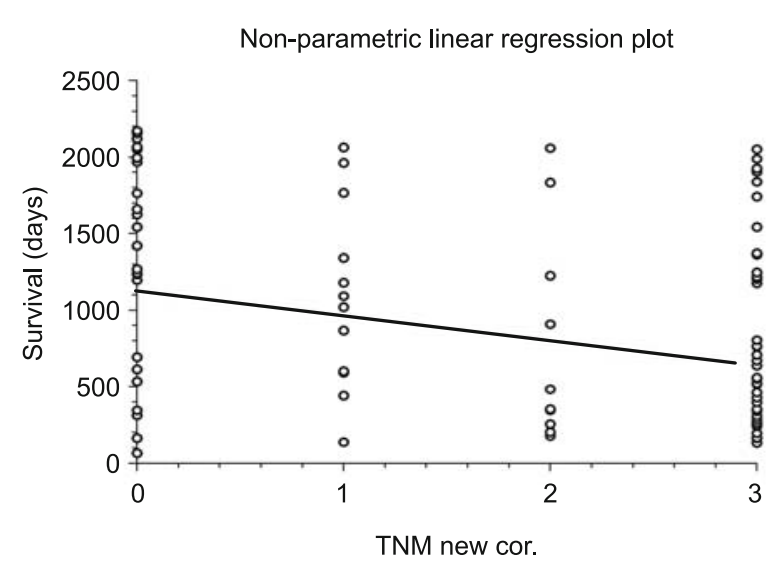

Fig. 8. Non-parametric correlation for 7th TNM.

Kendall sequential correlations

Survival-days vs N-ratio classification system

Observation of a sample $=119$

Kendall's tau b correlation coefficient $=-0.3343553$

Approximate $95 \% \mathrm{CI}=-0.4394892$ to -0.2292214

Correlation of the 7th TNM staging system vs survival-days (Fig. 8)

Non-parametric linear regression

Survival-days vs 7 th TNM

Observation of the sample $=85$

Median slope $(95 \% \mathrm{CI})=-162.75(-309$ to -42$)$

Y-intercept $=1128.5$

Kendall's tau b correlation coefficient $=-0.245884$

\section{Kendall sequential correlations}

Survival-days vs 7 th TNM classification system

Observation of a sample $=85$

Kendall's tau b correlation coefficient $=-0.245884$

Approximate $95 \% \mathrm{CI}=-0.380356$ to -0.111411

\section{Conclusion and discussion}

The existence of several staging systems has resulted in the emergence of various comparative studies and retrospective analyzes to evaluate the most appropriate staging system.

The survival of patients is evaluated according to different staging systems that are based on different parameters mentioned before.

Several studies confirmed the superiority of the 7th UICC Nstaging system to the 6th UICC N-staging classification in prognosis of overall survival (6).

In study from Italy, the staging systems based on the rate between the node counts was proved to be more reliable and accurate than the 7 th TNM in the prognostic stratification of gastric cancer patients (1).

In our work, we assumed that the comparison of survival of the same patient classified according to various systems answers the question of the importance of number vs anatomic location of affected lymph nodes (comparison of TNM vs JSGC).

In the first phase, we measured the survival in different groups of patients classified according to various staging systems (these are the same patients, only evaluated with the different classification systems except the patients excluded because of the specific criteria).

Due to the fact that while research has been in process, there was an update in 6th TNM classification to the 7th edition of TNM classification, patients were classified according to 7 th TNM as well. For the evaluation of the survival versus the time after operation, we used Kaplan Meier method (Figs 1 - 4).

The median survival, according to different staging systems, depending on the N-stage of the disease, we found a significantly correlated with survival ( $p<0.0001$ for 6 th TNM, $p=0.0002724$ for the JSGC, $\mathrm{p}=0.0000508$ for $\mathrm{N}$-ratio staging system, $\mathrm{p}=0.0129$ for the 7 th TNM).

The new finding was a differentiation of patients in the groups N1 vs N2 under the 6th TNM staging system $(\mathrm{HR}=0.910249)$, also a little differentiation in the groups N1 vs N2 according to the staging of $\mathrm{N}$-ratio $(\mathrm{HR}=0.8750926)$ and equally a poor differentiation survival in the groups N2 vs N3 according to the 7th TNM $(\mathrm{HR}=0.881797)$.

In order to assess, which staging system most reliably distinguishes patients according to their survival, based on the assumption that the best system is the one, according to which the classification depending on N-stage of the disease, has the most fundamental effect on survival. The question is whether we can tell where the statistical differences between NO, N1, N2 and N3 by individual classification systems significantly distinct. We conducted correlation using a parametric linear regression between the staging and the survival time that best differentiates patients. It should be taken into account that the individual analyzes are not identical in the number of patients. The most appropriate for this type of analysis in terms of design would be to do the analysis with the same patients in all staging systems.

The strongest correlation reached the Japanese classification system (tau $b=-0.385601$ ), but not significantly different from the 6th TNM (tau $b=-0.333416$ ). In the 7th TNM, we then found the weakest correlation with the survival time ( $\operatorname{tau} b=-0.245884$ ), but not significantly different from the previous two.

According to the results above, we can conclude that the patient survival significantly affected both the number of metastatic lymph nodes and their anatomical localization. The anatomical localization of the metastatic lymph nodes correlated with a better survival, but this difference was not confirmed as statistically significant.

We confirmed the strongest correlation between the patient's prognosis and the anatomic localization of metastatic lymph nodes in our study. However, this correlation was not statistically significant compared to the correlation between the patient's prognosis and the number of metastatic lymph nodes. We can therefore conclude that both classification systems are comparable and the difference is statistically insignificant. In future, probably a combination of the number and anatomic location of positive lymph nodes would be the solution to improve the stratification of patients 


\section{3-258}

with gastric cancer, allowing a better staging and then to indicate postoperative adjuvant therapy.

Well-designed randomized controlled trials are necessary to evaluate the most appropriate staging system.

\section{References}

1. Agnes A, Biondi A, Cananzi FM et al. Ratio-based staging systems are better than the 7th and 8th editions of the TNM in stratifying the prognosis of gastric cancer patients: A multicenter retrospective study. J Surg Oncol 2019: 1-10.

2. Deng J, Liang H, Wang D, Sun D, Ding X, Pan Y, Liu X. Enhancement the prediction of postoperative survival in gastric cancer by combining the negative lymph node count with ratio between positive and examined lymph nodes. Ann Surg Oncol 2010; 17: 1043-1051.

3. Cheong O, Oh ST, Kim BS et al. Large metastatic lymph node size, especially more than $2 \mathrm{~cm}$ : independent predictor of poor prognosis in node-positive gastric carcinoma. World J Surg 2008; 32: 262-266.

\section{Ielpo B, Pernaute AS, Elia S, Buonomo OC, Valladares LD, Aguirre} EP, Petrella G, Garcia AT. Impact of number and site of lymph node invasion on survival of adenocarcinoma of esophagogastric junction. Interact Cardiovasc Thorac Surg 2010; 10: 704-708.

5. Inoue K, Nakane $\mathbf{Y}$, Iiyama $\mathbf{H}$ et al. The superiority of ratio-based lymph node staging in gastric carcinoma. Ann Surg Oncol 2002; 9: 27-34.

6. Liu JY, Peng ChW, Yang XJ, Huang ChQ, Li Y. The prognosis role of AJCC/UICC 8th edition staging system in gastric cancer - a retrospective analysis. Am J Transl Res 2018; 10 (1): 292-303.

7. Marchet A, Mocellin S, Ambrosi A et al. The ratio between metastatic and examined lymph nodes (N-ratio) is an independent prognostic factor in gastric cancer regardless of the type of lymphadenectomy: results from an Italian multicentric study in 1853 patients. Ann Surg 2007; 245: 543-552.
8. Eskiler GG, Bezdegumeli E, Ozman Z, Devecki Ozkan A, Bilir C, Kucukakca BN, Ince MN, Men AY, Aktas O, Horoz YE, Akpinar D, Gene I, Kaleli S. IL-6 mediated JAK/STAT3 signaling pathway in cancer patients with cachexia. Bratisl Med J 2019; 120 (11): 819-826.

9. Konarikova K, Frivaldska J, Gbelcova H, Sveda M, Ruml T, Janubova M, Zitnanova I. Schiff base CU(11) complexes as inhibitors of proteasome in human cancer cells. Bratisl Med J 2019; 120 (9): 646-649.

10. Nitti D, Marchet A, Olivieri M, Ambrosi A, Mencarelli R, Belluco C, Lise M. Ratio between metastatic and examined lymph nodes is an independent prognostic factor after D2 resection for gastric cancer:analysis of a large european monoinstitutional experience. Ann Surg Oncol 2003; 10 (9): 1077-1085.

11. Pedrazzani C, Sivins A, Ancans G, Marrelli D, Corso G, Krumins V, Riviello F, Leja M. Ratio between metastatic and examined lymph nodes (N-ratio) may have low clinical utility in gastric cancer patients treated by limited lymphadenectomy: results from a single-center experience of 526 patients. World J Surg 2010; 34: 85-91.

12. Sayegh ME, Sano T, Dexter S, Katai H, Fukagawa T, Sasako M. TNM and Japanese staging systems for gastric cancer: how do they coexist? Gastric Cancer 2004; 7: 140-148.

13. Bernadic M, Jr, Duchon R, Aziri R, Mladosievicova B.: New principles of cancer therapy give new hope for oncological patients. Bratisl Med J 2019; 120 (1): 15-18.

14. Korourian A, Madjd Z, Roudi R, Shariftabrizi A, Soleimani M. Induction of miR-31 causes increased sensitivity to 5-FU and decreases migration and cell invasion in gastric adenocarcinoma. Bratisl Med J 2019; 120 (1): 35-39.

15. Smid D, Skalicky T, Dolezal J, Kubackova D, Fichtl J. Surgical treatment of gastric cancer. Bratisl Med J 2015; 116 (11): 666-670.

16. Toth $\mathbf{D}$, Biro A, Varga $\mathbf{Z}$ et al. Comparison of different lymph node staging systems in prognosis of gastric cancer: a bi-institutional study from Hungary. Chin J Cancer Res 2017; 29 (4): 323-332.

Received January 8, 2020. Accepted February 10, 2020. 